\title{
Clinical genetics evaluation in identifying the etiology of autism spectrum disorders: 2013 guideline revisions
}

\author{
G. Bradley Schaefer, MD'1 and Nancy J. Mendelsohn, MD² for the Professional Practice \\ and Guidelines Committee
}

\begin{abstract}
Disclaimer: This guideline is designed primarily as an educational resource for clinicians to help them provide quality medical services. Adherence to this guideline is completely voluntary and does not necessarily assure a successful medical outcome. This guideline should not be considered inclusive of all proper procedures and tests or exclusive of other procedures and tests that are reasonably directed to obtaining the same results. In determining the propriety of any specific procedure or test, the clinician should apply his or her own professional judgment to the specific clinical circumstances presented by the individual patient or specimen. Clinicians are encouraged to document the reasons for the use of a particular procedure or test, whether or not it is in conformance with this guideline. Clinicians also are advised to take notice of the date this guideline was adopted and to consider other medical and scientific information that becomes available after that date. It also would be prudent to consider whether intellectual-property interests may restrict the performance of certain tests and other procedures.
\end{abstract}

The autism spectrum disorders are a collective of conditions that have in common impaired socialization and communication in association with stereotypic behaviors. The reported incidence of autism spectrum disorders has increased dramatically over the past two decades. In addition, increased attention has been paid to these conditions by both lay and professional groups. These trends have resulted in an increase in the number of referrals to clinical geneticist for the evaluation of persons with autism spectrum disorders. The primary roles of the geneticist in this process are to define etiology when possible, to provide genetic counseling, and to contribute to case management. In deciding on the appropriate evaluation for a particular patient, the geneticist will consider a host of factors: (i) ensuring an accurate diagnosis of autism before proceeding with any investigation; (ii) discussing testing options, diagnostic yields, and family investment before proceeding with an evaluation; (iii) communicating and coordinating with the patient-centered medical home (PCMH); (iv) assessing the continuously expanding and evolving list of available laboratorytesting modalities in light of the published literature; (v) recognizing the expanded phenotypes of well-described syndromic and metabolic conditions that overlap with autism spectrum disorders; and (vi) defining an individualized evaluation plan based on the unique history and clinical features of a given patient. The guidelines in this paper have been developed to assist the clinician in the consideration of these factors. It updates the original publication from 2008.

Genet Med 2013:15(5):399-407

Key Words: Asperger syndrome; autism; diagnostic yield; pervasive developmental disorders; tiered evaluations
Autism spectrum disorders (ASDs), also known as pervasive developmental disorders, are a behaviorally defined group of neurodevelopmental disorders that are usually diagnosed in early childhood. They are characterized by varying degrees of limitations in communication and social interaction and by atypical, repetitive behaviors with an onset before 3 years of age. The phenotype of ASDs is extremely heterogeneous, with differences from person to person in a wide range of symptoms and severity as well as differences between the various subtypes of ASDs (e.g., autistic disorder, Asperger syndrome, and pervasive developmental disorder not otherwise specified).

Multiple lines of epidemiologic evidence support the strong role of genetics in the etiology of ASDs. ${ }^{1-3}$ Results of population studies of unselected cases of autism are most consistent with multifactorial inheritance. Until quite recently, the accepted recurrence risk for full siblings of a child with autism has been in the range of 3-10\%. ${ }^{4-6}$ Overall, only $2-3 \%$ of families have more than one affected child (possibly because of voluntary avoidance of pregnancy after a child is diagnosed). Most studies have reported a sex bias in the recurrence risk in keeping with the presumption of a "multifactorial" mode of inheritance (higher risk if the affected person is of the less commonly affected sex). As such, the reported risk is $7 \%$ of another affected child if the first affected child is female and $4 \%$ if the first affected child is male. ${ }^{7}$ If multiple children (two or more) have autism, the recurrence risk is on the order of $33-50 \%$ for any future pregnancy. ${ }^{7}$

Two recent studies ${ }^{8,9}$ have reported even higher recurrence risks of 11 and $19 \%$ with single-sibling involvement. The first ${ }^{8}$ was a retrospective self-enrolled/self-identified study using an interactive website. The diagnosis was confirmed, but the identification of second siblings may be a source of ascertainment bias. The second ${ }^{9}$ was an international multisite prospective study in 664 families with a calculated 19\% recurrence risk. Interestingly, the typically reported sex bias was not noted in one of these studies. ${ }^{8}$ Both were single studies that bear replication. 
At present, it is not suggested that these numbers be used in modifying recurrence-risk guidelines for genetic counseling.

The roles of the clinical geneticist are to determine the etiology of the ASD when possible, to improve care and management, and to provide genetic counseling for the family. In recent years, there has been an increase in new diagnostic options available to the clinician. Several publications have also presented many "expanded phenotypes" for genetic and metabolic conditions in association with ASDs' phenotypes. ${ }^{10-15}$ These factors have led to an increase in the number of referrals to the clinical geneticist and an increase in the diagnostic yield.

In deciding on an evaluation plan, the clinical geneticist has the task of balancing an ever-expanding list of available tests and possible diagnoses with the issues of cost, practicality, and expected yield. These considerations need to be further balanced with the possible benefits of a diagnosis such as treatment opportunities, improved outcomes, and specific recurrence-risk information for the family. The guidelines put forth here outline a tiered evaluation of the etiology of ASDs based on current evidence. Most of the evidence cited is from medium-sized to large case series, but some is from larger population-based studies.

\section{RATIONALE FOR AN EVALUATION}

The rationale for a genetic evaluation is based on the goal of identifying a unifying diagnosis for a patient. Clinical geneticists can contribute to the process by examining and evaluating the patient, his or her parents, and siblings as necessary in establishing the etiology. A definitive diagnosis facilitates acquisition of needed services and is helpful in many other ways for the family. Many families are greatly empowered by knowing the underlying cause of a relative's disorder. Depending on the etiology, associated medical risks may be identified that lead to screening and the potential for prevention of morbidity. Specific recurrence-risk counseling-beyond general multifactorial information - can be provided, and targeted testing of at-risk family members can be offered. Finally, an established diagnosis will help in eliminating unnecessary diagnostic tests. In light of these expected benefits, a genetic evaluation should be offered to every person with an ASD (or his or her family).

\section{REPORTED APPROACHES AND YIELDS}

The rate of success for identifying a specific etiologic diagnosis in persons with ASDs has been reported as 6-15\%. ${ }^{16-20}$ This range is applicable even for evaluations of patients with pervasive developmental disorder not otherwise specified, atypical autism, Asperger syndrome, or autistic features who did not necessarily meet the criteria of the fourth edition of the Diagnostic and Statistical Manual of Mental Disorders criteria for pervasive developmental disorder. ${ }^{21}$

Many factors seem to influence the diagnostic yield. Not surprisingly, the clinical skills and experience of the medical geneticist factor into the likelihood of defining a cause. Clearly, advances in testing modalities improve the diagnostic yield..$^{22} \mathrm{~A}$ review of the potential contribution of newer testing modalities (presented below) can be expected to be between 30 and $40 \%$.
In the following paragraphs, we provide the results of diagnostic yields for specific types of tests. Caution must be exercised in interpreting such studies across times. Because the reported incidence of autism has increased, it is possible that the relative proportion of cases attributable to a given etiology has changed. ${ }^{23}$ Likewise, the rapid rate of change in testing technologies may complicate comparisons. For instance, the various types of chromosomal microarray (CMA)-bacterial artificial chromosome, oligonucleotide, or single-nucleotide polymorphism arrays-are not necessarily comparable in the type and quantity of their diagnostic yields.

\section{Chromosomal abnormalities}

Chromosomal abnormalities have consistently been reported in persons with ASDs. Continued improvements in cytogenetic approaches, including higher-resolution studies, have increased the diagnostic yield of conventional cytogenetic studies to approximately $3 \%{ }^{24-26}$ Numerous cytogenetically detectable deletions and duplications have been associated with an ASD phenotype. ${ }^{27}$ In general, the most commonly reported loci mirror the reported linkage data. Some of the most frequently reported regions are listed in Table 1. The advent of molecular cytogenetic testing modalities such as CMA has improved the diagnostic power of genetic evaluations (see below). This progress has been so effective that it has largely replaced conventional cytogenetics as a first-tier test. ${ }^{28,29}$ For this reason, chromosomal analysis in the evaluation of persons with ASDs should now be reserved only for certain exceptions such as a clinically suspected chromosome aneuploidy (e.g., Turner, Klinefelter, and Down syndromes) or a family or reproductive history suggestive of chromosomal rearrangements. There are still situations in which third-party payers will cover cytogenetic studies but not CMA testing. In such a situation (and when the patient cannot afford the cost of the CMA studies), a conventional chromosomal analysis is preferable to no cytogenetic testing at all.

\section{Chromosomal microarray}

CMA has emerged as a powerful tool for clinical genetic testing. ${ }^{29,30}$ Currently, two methods of CMA are used in the clinical setting. Array-comparative genomic hybridization and single-nucleotide polymorphism arrays use different techniques to scan the genome for copy-number variants (CNVs). With the increased number of CMA studies, new information has emerged regarding the contribution of genome CNVs in ASDs. Estimates of $\mathrm{CNV}$ frequencies in unselected populations of individuals with an ASD are from 8 to $21 \%$. One of these studies was reported in $2007 .{ }^{31}$ The other five were recent (2010 and 2011) studies and used high-density arrays. Cumulatively, these six independent studies of 2,805 patients with ASDs found 274 (10\%) with CNVs that were reported as being clinically significant. ${ }^{31-36}$

More than 100 different genomic changes have been reported in individuals with ASDs. ${ }^{37}$ Among these, several have been shown to have an especially strong association. Not surprisingly, 
Table 1 Selected genetic "hot spots" in autism as identified by selected testing modalities

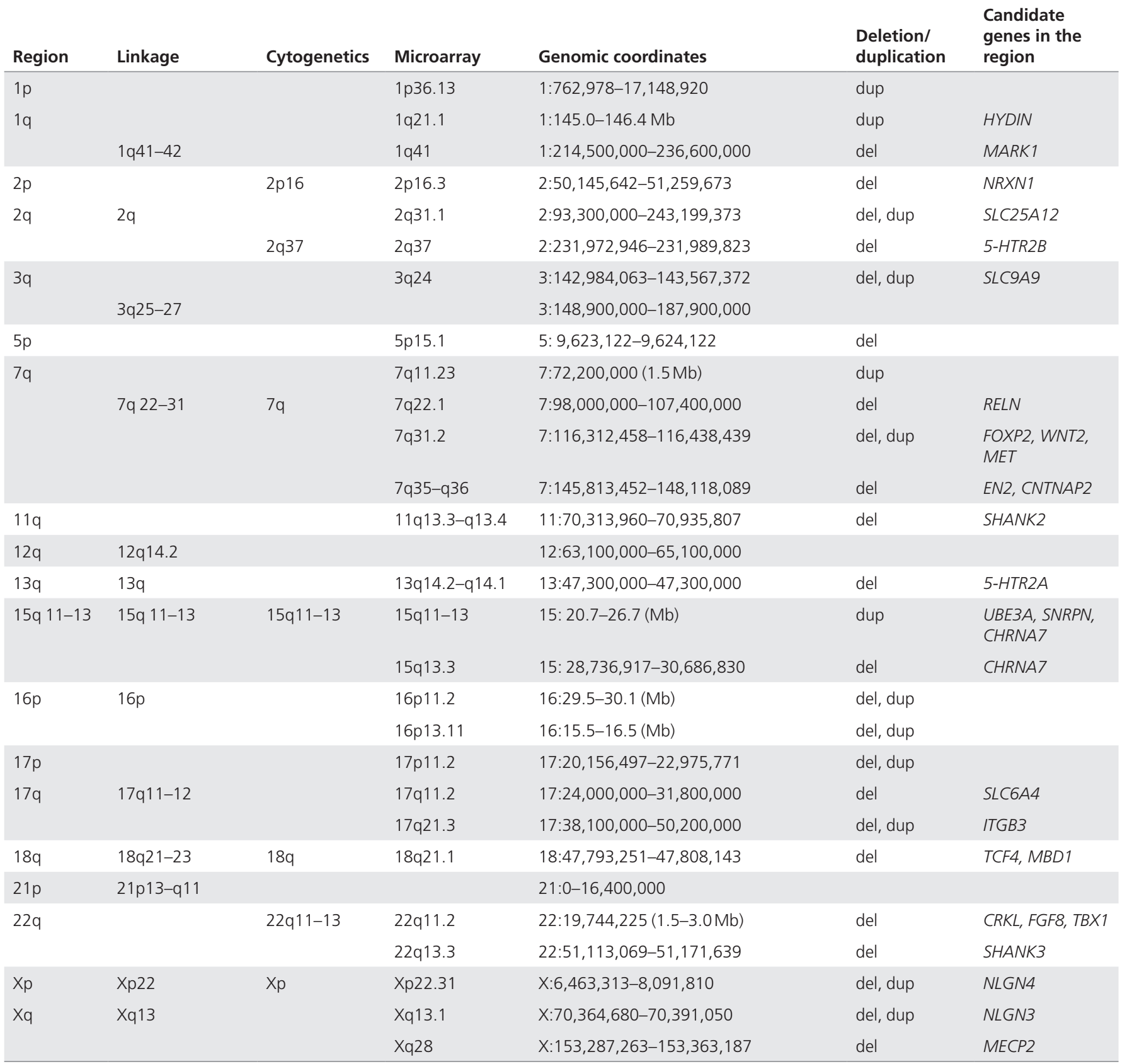

some of these "ASD hot spots" correlate with areas highlighted by earlier linkage and cytogenetic studies. The most commonly noted ASD loci on CMA studies are listed in Table 1. Some of these loci can be quite common in an ASD population. In fact, one locus (16p11.2) has been reported to have CNVs occurring in $0.5-1 \%$ of all individuals with ASDs. ${ }^{38-41}$ Given this diagnostic yield, we have moved CMA to a first-tier test in place of a karyotype, as suggested in the 2008 guidelines. This is in keeping with recent consensus opinion that CMA is a first-tier test for individuals with developmental disabilities or congenital anomalies. ${ }^{29}$

The diagnostic yield of CMA studies in patients with ASDs can be increased using selection criteria. Higher rates of CNVs are noted in individuals with ASDs and additional findings such as microcephaly, seizures, congenital anomalies, or dysmorphic features (so-called "complex ASDs"). Performing CMA studies only in individuals with complex ASDs has been shown to increase the diagnostic yield to almost $30 \%{ }^{33,42}$ Another selection factor that can increase the yield of CMA studies in individuals with ASDs is a family history of developmental disabilities or psychiatric problems.

Once a CNV has been identified by CMA in a patient with autism, there are several critical considerations in interpreting the information and conveying it to the family in a meaningful format. Copy-number changes show marked intra- and 
interfamilial variability in their clinical manifestations. The range of variable expression can include individuals with cognitive and social abilities within the normal range. Therefore, in determining whether such a change is de novo or potentially inherited, parental studies should be offered, even in the absence of apparent neurobehavioral disorders in the parents. Moreover, careful attention should be paid to the family history with emphasis placed on other neurodevelopmental and neurobehavioral disorders. The identical CNV seen in a person with an ASD might present as neurologic symptoms (such as seizures), attention-deficit hyperactivity disorder, intellectual disability, or a major affective disorder in a relative. All of the complexities of changing technology, dynamically expanding literature, and large accessible informational databases can make genetic counseling challenging even when such an etiologic diagnosis is made. The geneticist and genetic counselor must be sure to note the type of array technology used (e.g., BAC vs. oligonucleotide vs. single-nucleotide polymorphism arrays) and to access major data resources such as the International Standards for Cytogenomic Arrays consortium, ${ }^{43}$ the UCSC Human Genome Browser, ${ }^{44}$ DECIPHER (Database of Chromosomal Imbalance and Phenotype in Humans Using Ensembl Resources), ${ }^{45}$ and the Database of Genomic Variants ${ }^{46}$ in order to provide the best possible information for the family.

\section{Single-gene disorders}

Several well-described single-gene disorders have been reported for which ASDs can be seen as part of the expanded phenotype associated with changes in that gene (Table 1). For a selected few of such conditions, there is adequate evidence to suggest testing for changes in these genes in patients with ASDs with no other identifiable etiology. These would include fragile $\mathrm{X}$ syndrome, methyl-CPG-binding protein 2 (MECP2) spectrum disorders, and phosphatase and tensin homolog (PTEN)related conditions.

There is a long-standing association of ASDs with fragile $\mathrm{X}$ syndrome. Approximately $20 \%$ of boys with fragile X syndrome meet diagnostic criteria for ASDs when evaluated by objective criteria. ${ }^{11,47}$ Two early studies found an incidence of abnormal fragile X studies of $6 \%$ in unselected cohorts of boys with ASDs. These studies noted 4 of $75(5.3 \%)^{48}$ and 17 of $210(8 \%)^{49}$ positive rates. Recent surveys have reported a lower yield for fragile $\mathrm{X}$ testing. Three recent studies identified fragile $\mathrm{X}$ in only 6 of $1,215(0.5 \%)$ children with ASDs. ${ }^{22,34,36}$ It seems likely that some of these differences can be explained by better recognition and earlier diagnosis of fragile $\mathrm{X}$ syndrome. Once this diagnosis is made, many centers will not assign these children to ASDs clinics; therefore, they may not be included in overall "unselected" studies. It is recommended that all males with unexplained autism be tested for fragile $\mathrm{X}$ syndrome.

Our review of the literature did not identify any female patients with abnormal fragile $\mathrm{X}$ results from among an unselected cohort of persons with ASDs. Therefore, "routine" testing of females with ASDs for fragile X does not meet evidence-based criteria. However, we recommend that serious consideration be given to ordering fragile $\mathrm{X}$ studies in females with ASDs when prompted by clinical parameters such as (i) a phenotype compatible with fragile X; (ii) a family history positive for X-linked neurodevelopmental disorders; or (iii) premature ovarian insufficiency, ataxia, or tremors in close relatives.

Mutations in MECP2 were originally reported as the primary etiology of Rett syndrome. It was not long until a broad range of other phenotypes were described in conjunction with MECP2 mutations. Idiopathic ASD was one such phenotype. ${ }^{50}$ The aggregate data from nine studies ${ }^{21,22,51-57}$ in which MECP2 testing was performed on girls with nonsyndromic ASDs identified 16 of $400(4 \%)$ with pathogenic mutations (range: $0-20 \%)$. Interestingly, in one of these studies, ${ }^{54}$ the only patients identified with a mutation were those with a phenotype of the "preserved speech variant" subtype of Rett syndrome. An additional study ${ }^{58}$ drew from two large databases to evaluate the presenting complaint of 313 cases of girls with MECP2 mutations. The authors found that the patients with an initial diagnosis of autism had fewer symptoms of classic Rett syndrome and better functional outcomes. The girls with an autism diagnosis and MECP2 mutations were associated with two specific sequencing mutations: R306C and T158M. In 2008, when the original guidelines were written, no males with MECP2 abnormalities and autism had been reported. Since then, two publications ${ }^{59,60}$ have identified males with $M E C P 2$ duplications and an ASD phenotype. Among the four studies, 22 males with known MECP2 mutations were evaluated. Of these, nine had a diagnosis of autism and one was noted to have "autistic features." Clearly, these are small studies and were not designed to assess the prevalence in an unselected ASD population. Given current evidence, routine $M E C P 2$ testing in males with autism is not recommended. However, the geneticist should be alert to the features of $M E C P 2$ duplications (e.g., drooling, recurrent respiratory infections, hypotonic facies) and consider MECP2 duplication testing in boys with autism and such features.

In 2001, a mother and her son were found to have identical PTEN mutations. ${ }^{61}$ The phenotype in the mother was Cowden syndrome. The child, too, had Cowden syndrome but also had an ASD. The authors raised the question of a possible association. A few years later, a series of 18 patients selected for ASDs with macrocephaly (head circumference $>2.5$ SDs above the mean) was reported..$^{10}$ Of these, three patients (16\%) had PTEN mutations. Other single-case reports of ASDs in conjunction with PTEN mutations have appeared in the literature. At least one of these individuals was female. ${ }^{62}$ In addition to the previously mentioned studies, four other reported studies have looked at PTEN mutations in cohorts of persons with ASDs. ${ }^{56,63-65}$ The collective data from these studies identified 15 of 318 individuals (5\%) with ASDs who had pathogenic PTEN mutations. Although most of these studies did not select for patients with ASDs with macrocephaly, retrospective analysis showed that macrocephaly was present in all positive cases. Therefore, it is suggested that PTEN testing be reserved for patients with ASDs with a head circumference above the 98th percentile. It 
is important to consider parental head sizes as well as the pattern of head growth in determining whether the head size is truly excessive or whether a parent may be mildly affected. Also highlighted by these studies, the yield may be exceptionally high in patients with ASDs and macrocephaly of $>4$ SDs above the mean.

When a family history is consistent with X-linked inheritance and the patient has cognitive impairments, an "X-linked intellectual disability gene panel" is a consideration. Several X-linked genes are known to present as either ASD or intellectual disability. ${ }^{37}$ Another disorder to consider is the X-linked creatine transporter defect (SCL6A8 gene). Patients with this condition have been reported with neurobehavioral changes in the ASD spectrum, along with hypotonia and seizures. Currently, no studies have been reported on the diagnostic yield of such panels in persons with ASDs.

Table 2 Recently described metabolic conditions associated with an ASD phenotype

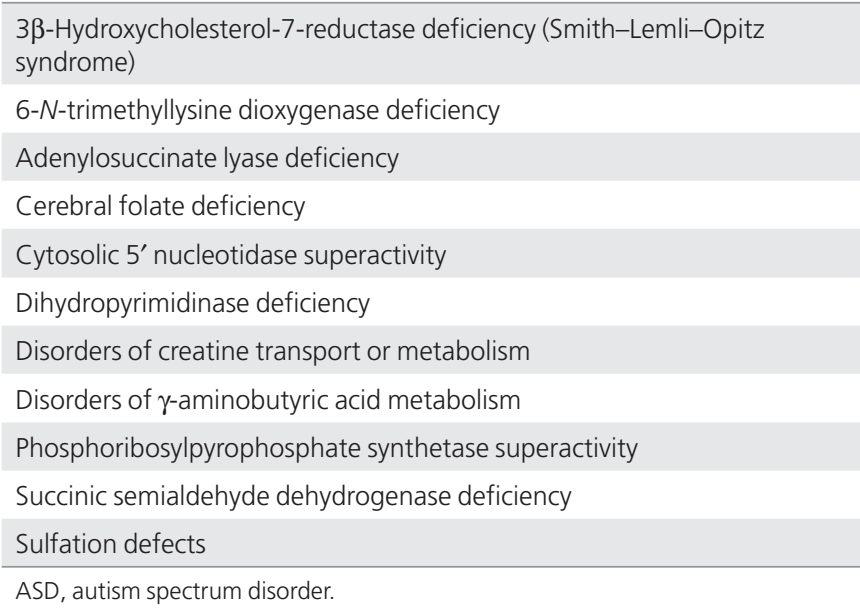

Table 3 Clinical symptoms that prompt metabolic or mitochondrial testing in persons with ASDs

Acid/base or electrolyte disturbances

Anemia with an elevated mean corpuscular volume

Cyclic vomiting

Dermatologic changes: alopecia, hypertrichosis, and pigmented skin eruptions

Developmental regression associated with illness or fever

Gastrointestinal dysfunction, gastroparesis

Hypotonia/dystonia

Lactic acidosis

Lethargy

Multisystem involvement, especially cardiac, hepatic, or renal (physical and/or laboratory evidence)

Neurodegeneration outside of the typical ASD speech loss at 18-24 months

Poor growth, microcephaly

Seizures

ASD, autism spectrum disorder.

\section{Metabolic disorders}

Numerous metabolic abnormalities have been reported in the context of an ASD phenotype (Table 2). Metabolic disorders associated with an ASD phenotype are relatively rare, typically autosomal-recessive in nature, and present early in life. ${ }^{66}$ Most metabolic disorders are associated with other clinical symptomatology such as seizures, extrapyramidal signs (movement disorders, dystonia, and parkinsonism), failure to thrive, or neuroregression that is atypical for the autistic patient. To our knowledge, there have been no systematic studies that have looked at the diagnostic yield of metabolic testing in an unselected cohort of patients with ASDs. A joint plenary session of the American College of Medical Genetics and the Society for Inherited Metabolic Disorders in 2009 addressed this issue. The general opinion expressed was that metabolic disorders in ASDs were indeed "low incidence yet high impact." Although no consensus was reached on what level of testing should be recommended, several important themes were highlighted. Similar to a clinician's responsibility to evaluate a patient with ASD for syndromic features, the geneticist should review the patient's history for signs and symptoms that would warrant further metabolic evaluation or referral (Table 3). A careful medical history for true developmental regression (neurodegeneration), worsening neurological symptoms, lethargy, poor physical endurance, or seizures is critical. Physiologic abnormalities such as acidosis should be aggressively pursued. Although this guideline does not recommend routine metabolic testing in the absence of clinical indicators, the index of suspicion for inborn errors of metabolism must remain high. For some metabolic disorders, there may be no clinical indicators. For instance, it has been reported that $5 \%$ of patients with untreated phenylketonuria will meet diagnostic criteria for autism. ${ }^{67}$ Likewise, the clinical indicators for Sanfilippo syndrome may not be apparent for decades after the diagnosis of autism. ${ }^{68} \mathrm{~A}$ procedural question has been raised in this context: how would the geneticist know whether acidosis was present if testing were not performed in the first place? Hence, many metabolic specialists have suggested that routine metabolic screening of patients with ASDs be performed as a first-tier evaluation. Such an evaluation would include a complete blood count, a serum metabolic profile, and serum amino acid and urine screening for glycosaminoglycans. As for many of these unresolved issues, a prospective study is needed to identify the actual yield of such suggested screenings.

\section{Mitochondrial abnormalities}

New literature suggests a link between mitochondrial dysfunction and ASDs. ${ }^{69}$ This association has been recognized in persons with autistic behaviors and loss of speech after a febrile illness or immunization with subsequent encephalopathy. ${ }^{70}$ Constitutional symptoms, hypotonia, repeated regressions after the age of 3 years, and multiple organ dysfunctions are clues to consider mitochondrial disease (Table 3). A review of 25 patients with a known mitochondrial disorder who presented with an initial diagnosis of ASD found that all had an abnormal 
neurologic examination and/or an elevated plasma lactate concentration. ${ }^{70}$ As with metabolic disorders, testing for mitochondrial disorders in persons with ASDs is recommended only if supporting symptoms or laboratory abnormalities are present.

\section{Neuroimaging}

The role of neuroimaging in the evaluation of persons with developmental disabilities has been strongly debated for decades. To some, it is intuitive that one should "examine" the affected organ. Others argue that cost and the logistics of sedation make such studies too risky for the yield. It has also been suggested that multiple sedations at an early age are associated with adverse neurodevelopmental outcomes. ${ }^{71,72} \mathrm{~A}$ fairly recent publication $^{73}$ reported a $48 \%$ (33 of 69) occurrence of identifiable brain anomalies in an unselected cohort of persons with ASDs. However, most identified abnormalities were not diagnostic of a specific condition. Currently, there is insufficient evidence to recommend routine magnetic resonance imaging scans for all patients with ASDs. In the event of important comorbid conditions such as neuroregression or other major neurologic abnormalities, magnetic resonance imaging can be a helpful diagnostic tool. One special situation also warrants mentioning. In contrast to macrocephaly, microcephaly and autism are rarely associated. It is also important to note whether the microcephaly is congenital or acquired, because the differential diagnosis differs. Neuroimaging should be considered in individuals with ASDs and microcephaly. For patients in whom a metabolic etiology is suspected, combining magnetic

\section{Table 4 Template for the clinical genetic diagnostic evaluation of autism spectrum disorder}

\section{First tier}

Three-generation family history with pedigree analysis

Initial evaluation to identify known syndromes or associated conditions

Examination with special attention to dysmorphic features

If specific syndromic diagnosis is suspected, proceed with targeted testing

If appropriate clinical indicators present, perform metabolic and/ or mitochondrial testing (alternatively, consider a referral to a metabolic specialist)

Chromosomal microarray: oligonucleotide array-comparative genomic hybridization or single-nucleotide polymorphism array

DNA testing for fragile $X$ (to be performed routinely for male patients only) $)^{a}$

Second tier

MECP2 sequencing to be performed for all females with ASDs

MECP2 duplication testing in males, if phenotype is suggestive

PTEN testing only if the head circumference is $>2.5 \mathrm{SD}$ above the mean

Brain magnetic resonance imaging only in the presence of specific indicators (e.g., microcephaly, regression, seizures, and history of stupor/coma)

ASD, autism spectrum disorder; MECP2, methyl-CPG-binding protein 2; PTEN , phosphatase and tensin homolog.

aDNA testing for fragile $X$ in females if indicators present (e.g., family history and phenotype). resonance spectroscopy with standard neuroimaging should be considered.

\section{Summary}

In summary, the literature indicates that the following approximate diagnostic yields are expected in the genetic evaluation of ASDs:

- $\operatorname{CMA}(10 \%)$

- Fragile X (1-5\%)

- MECP2 (4\% of females)

- PTEN (5\% of those with head circumferences $>2.5$ SDs that are tested)

- Karyotype (3\%)

- Other (10\%). Currently, there are no published studies that collate the yield on the other identifiable etiologies of autism. As noted above, identifiable brain anomalies, genetic syndromes, metabolic disorders, and other diagnosable conditions will be identified in the genetic evaluation of persons with ASDs. Using empiric estimates and clinical experience, this has been estimated as $10 \%{ }^{74}$

Therefore, using current knowledge and technology, a thorough clinical genetics evaluation of patients with ASDs is estimated to result in an identified etiology in $30-40 \%$ of individuals.

\section{EVALUATION}

The clinical geneticist's major contributions continue to be identification of dysmorphic features and analysis of family-history information. Clinically recognizable syndromes that may be subtle are another category of identifiable causes of ASDs. ${ }^{75}$ Therefore, an evaluation by a clinical geneticist/dysmorphologist to determine whether an individual with ASD has isolated or complex/syndromic ASD remains a high yield, low-cost part of the evaluation.

Like all clinical evaluations, an etiologic evaluation must be tailored to the individual patient. The design of the evaluation must take into consideration focused information from the history and the physical examination as well as clinical experience. There is no single approach or algorithm that can be applied to all cases. For practical reasons, a stepwise, or tiered, evaluation is recommended in this report (Table 4). A stepwise evaluation can be designed such that tests performed in higher (earlier) tiers have a greater expected diagnostic yield, lower invasiveness of testing, and better potential for intervention, and are more easily employed. ${ }^{76}$

This approach will evolve with continued advancements in diagnostic testing and improved understanding of the ASD phenotype. Multiple additional conditions have been reported in association with an ASD phenotype, but none of these has been evaluated in a large prospective cohort. Therefore, a future third tier of evaluation is a distinct possibility. Further studies would be needed to elevate the evidence to the point of recommended testing. Alternatively, advances in technology may 
permit bundling of individual tests into an extended, more readily accessible, and less expensive platform.

\section{THE DIAGNOSTIC PROCESS}

1. Accurate diagnosis: It is critical that an accurate diagnosis of ASD be made before initiating the genetic evaluation. All patients with ASDs should have a formal audiogram to rule out a significant hearing loss.

2. Role of the PCMH: Every individual with an ASD should have a designated primary-care medical home. Often, the primary care physician will be the first professional to raise the question of ASD as a possible diagnosis. After clinical genetics consultation, the primary care physician and the clinical geneticist should be prepared to partner in ordering, scheduling, and coordinating recommended diagnostic tests.

3. Referral for clinical genetics evaluation: A genetic consultation should be offered to all persons/families with ASDs. Evaluations should be considered for any individual along the entire ASDs spectrum. The referring professional should discuss expectations and possible outcomes of such an evaluation before making the referral. The referring professional should be aware of what is involved in such a consultation and the potential diagnostic yields and should share this information with the patient/family.

4. Tiered evaluation: The clinical genetic evaluation of an individual with an ASD must be customized to the clinical situation. A patient may be referred to the geneticist with the goal of confirming a specific diagnosis that is being considered. Alternatively, a syndromic diagnosis may be apparent to the geneticist in the initial visit. In either case, the diagnosis should be confirmed using accepted clinical criteria and/or laboratory testing (if available). Many recognizable syndromes have a firmly documented association with

Table 5 Selected genetic syndromes that are known etiologies of autism spectrum disorders

22q11.2 deletions including velocardiofacial (Shprintzen) syndrome

Angelman syndrome

CHARGE syndrome

de Lange syndrome

Fragile $X$ syndrome

MED12 disorders (including Lujan-Fryns syndrome)

Prader-Willi syndrome

PTEN-associated disorders (Cowden syndrome, Bannayan-Riley-

Ruvalcaba syndrome)

Rett syndrome

Smith-Lemli-Opitz syndrome

Smith-Magenis syndrome

Sotos syndrome

Tuberous sclerosis

PTEN, phosphatase and tensin homolog.

Adapted from ref. 74.
ASDs. For these conditions, further investigation into the etiology of the ASD is unnecessary (Table 5).

There are, however, genetic conditions that have been reported in association with ASDs for which the reported association is not convincing. For patients with these conditions, it is recommended that an etiologic evaluation of the ASD be undertaken as an independent process (Table 6). If the clinical geneticist does not identify a specific disorder in the initial evaluation, further testing can be accomplished as outlined in Table 4.

5. Genetic counseling: Upon completion of the clinical genetics evaluation, two groups of individuals will have been identified: those with and those without an identifiable etiology. Genetic counseling should be provided to both groups. For those without an identifiable etiology, counseling should be provided using empiric recurrence-risk data. The accepted published recurrence risk for full siblings is approximately $3-10 \%$-although newer studies are suggesting that this risk may be higher. ${ }^{8,9}$ Modified for sex, the risks are $7 \%$ if the affected child is female and $4 \%$ if the affected child is male. If there are multiple children (two or more) with ASDs, published reports would predict at least a $30 \%$ recurrence risk. ${ }^{7,9}$

6. Treatment and follow-up: Clinical geneticists differ greatly in their practice as to their involvement with patients after completion of diagnostic consultations. Management and treatment plans depend on specific etiologic diagnoses. Such cases are often comanaged by agreement by the clinical geneticist and the primary care physician. Changes in technology and in phenotypes often aid in ultimately obtaining a diagnosis in patients for whom a diagnosis is not initially established. Thus, periodic reevaluations should be considered for patients in whom a definitive etiology is not initially discovered. The timing of interval follow-up consultations should be negotiated among the patient/family, the PCMH, and the medical geneticist.

Table 6 Genetic tests that have been suggested in the etiologic evaluation of ASDs, but currently with insufficient evidence to recommend routine testing

CDLK5 testing

Cholesterol/7 dehydrocholesterol

Chromosome 15 methylation/UBE3A gene testing

Methylation/epigenetic testing

Mitochondrial gene sequencing/oligoarray

NSD1 testing

Reduction-oxidation studies

Screening for disorders of purine/pyrimidine metabolism (serum and urine uric acid)

Screening for folate-sensitive fragile sites

Selected neurometabolic screening (mucopolysaccharides, creatinine phosphokinase, amino acids, organic acids, lactate, ammonia, acylcarnitine profile)

ASD, autism spectrum disorder. 


\section{Summary}

A genetic basis for autism is strongly supported by a large body of literature. Advances in clinical testing technology have increased the diagnostic yield from $6-10 \%$ a few years ago to $30-40 \%$. Therefore, genetic testing should be discussed with all patients and families with ASDs. This document provides recommendations on how to proceed with such an investigation. It is also important to note the accumulating evidence that newer testing modalities applying "next-generation" sequencing such as exome or whole-genome sequencing approaches will increase the diagnostic yield even more over the next few years.

\section{DISCLOSURE}

The authors declare no conflict of interest.

\section{REFERENCES}

1. El-Fishawy P, State MW. The genetics of autism: key issues, recent findings, and clinical implications. Psychiatr Clin North Am 2010;33:83-105.

2. Geschwind DH. Genetics of autism spectrum disorders. Trends Cogn Sci (Regul Ed) 2011;15:409-416.

3. Caglayan AO. Genetic causes of syndromic and non-syndromic autism. Dev Med Child Neurol 2010;52:130-138.

4. Chakrabarti S, Fombonne E. Pervasive developmental disorders in preschool children. JAMA 2001;285:3093-3099.

5. Icasiano F, Hewson P, Machet P, Cooper C, Marshall A. Childhood autism spectrum disorder in the Barwon region: a community based study. J Paediatr Child Health 2004;40:696-701.

6. Lauritsen MB, Pedersen CB, Mortensen PB. Effects of familial risk factors and place of birth on the risk of autism: a nationwide register-based study. J Child Psychol Psychiatry 2005;46:963-971.

7. Simonoff E. Genetic counseling in autism and pervasive developmental disorders. J Autism Dev Disord 1998;28:447-456.

8. Constantino JN, Zhang Y, Frazier T, Abbacchi AM, Law P. Sibling recurrence and the genetic epidemiology of autism. Am J Psychiatry 2010;167:13491356.

9. Ozonoff $S$, Young GS, Carter A, et al. Recurrence risk for autism spectrum disorders: a Baby Siblings Research Consortium study. Pediatrics 2011;128:e488-e495.

10. Butler MG, Dasouki MJ, Zhou XP, et al. Subset of individuals with autism spectrum disorders and extreme macrocephaly associated with germline PTEN tumour suppressor gene mutations. J Med Genet 2005;42: 318-321.

11. Clifford S, Dissanayake C, Bui QM, Huggins R, Taylor AK, Loesch DZ. Autism spectrum phenotype in males and females with fragile $X$ full mutation and premutation. J Autism Dev Disord 2007;37:738-747.

12. Erlandson A, Hagberg B. MECP2 abnormality phenotypes: clinicopathologic area with broad variability. J Child Neurol 2005;20:727-732.

13. Morrow JD, Whitman BY, Accardo PJ. Autistic disorder in Sotos syndrome: a case report. Eur J Pediatr 1990;149:567-569.

14. Park JP, Moeschler JB, Davies WS, Patel PI, Mohandas TK. Smith-Magenis syndrome resulting from a de novo direct insertion of proximal $17 q$ into 17p11.2. Am J Med Genet 1998;77:23-27.

15. Pearl PL, Gibson KM, Acosta MT, et al. Clinical spectrum of succinic semialdehyde dehydrogenase deficiency. Neurology 2003;60:1413-1417.

16. Chudley AE, Gutierrez E, Jocelyn LJ, Chodirker BN. Outcomes of genetic evaluation in children with pervasive developmental disorder. J Dev Behav Pediatr 1998;19:321-325.

17. Steiner CE, Guerreiro MM, Marques-de-Faria AP. Genetic and neurological evaluation in a sample of individuals with pervasive developmental disorders. Arq Neuropsiquiatr 2003;61(2A):176-180.

18. Challman TD, Barbaresi WJ, Katusic SK, Weaver A. The yield of the medical evaluation of children with pervasive developmental disorders. J Autism Dev Disord 2003;33:187-192.

19. Kosinovsky B, Hermon $S$, Yoran-Hegesh $R$, et al. The yield of laboratory investigations in children with infantile autism. J Neural Transm 2005;112:587596.
20. Battaglia A, Carey JC. Etiologic yield of autistic spectrum disorders: a prospective study. Am J Med Genet C Semin Med Genet 2006;142C:3-7.

21. Abdul-Rahman $O A$, Hudgins $L$. The diagnostic utility of a genetics evaluation in children with pervasive developmental disorders. Genet Med 2006;8:50-54.

22. Roesser J. Diagnostic yield of genetic testing in children diagnosed with autism spectrum disorders at a regional referral center. Clin Pediatr (Phila) 2011;:50:834-843.

23. Lathe R. Fragile X and autism. Autism 2009;13:194-197.

24. Reddy KS. Cytogenetic abnormalities and fragile-X syndrome in autism spectrum disorder. BMC Med Genet 2005;6:3.

25. Shevell MI, Majnemer A, Rosenbaum P, Abrahamowicz M. Etiologic yield of autistic spectrum disorders: a prospective study. J Child Neurol 2001;16:509512.

26. Weidmer-Mikhail E, Sheldon S, Ghaziuddin M. Chromosomes in autism and related pervasive developmental disorders: a cytogenetic study. J Intellect Disabil Res 1998;42(Pt 1):8-12.

27. Vorstman JA, Staal WG, van Daalen $E$, van Engeland $H$, Hochstenbach PF, Franke L. Identification of novel autism candidate regions through analysis of reported cytogenetic abnormalities associated with autism. Mol Psychiatry 2006;11:1,18-28.

28. Manning M, Hudgins L; Professional Practice and Guidelines Committee. Array-based technology and recommendations for utilization in medical genetics practice for detection of chromosomal abnormalities. Genet Med 2010;12:742-745.

29. Miller DT, Adam MP, Aradhya S, et al. Consensus statement: chromosomal microarray is a first-tier clinical diagnostic test for individuals with developmental disabilities or congenital anomalies. Am J Hum Genet 2010;86:749-764.

30. Coulter ME, Miller DT, Harris DJ, et al. Chromosomal microarray testing influences medical management. Genet Med 2011;13:770-776.

31. Sebat J, Lakshmi B, Malhotra D, et al. Strong association of de novo copy number mutations with autism. Science 2007;316:445-449.

32. Rosenfeld JA, Ballif BC, Torchia BS, et al. Copy number variations associated with autism spectrum disorders contribute to a spectrum of neurodevelopmental disorders. Genet Med 2010;12:694-702.

33. Schaefer GB, Starr L, Pickering D, Skar G, Dehaai K, Sanger WG. Array comparative genomic hybridization findings in a cohort referred for an autism evaluation. J Child Neuro/ 2010:25:1498-1503.

34. Shen Y, Dies KA, Holm IA, et al.; Autism Consortium Clinical Genetics/DNA Diagnostics Collaboration. Clinical genetic testing for patients with autism spectrum disorders. Pediatrics 2010;125:e727-e735.

35. Bremer A, Giacobini M, Eriksson M, et al. Copy number variation characteristics in subpopulations of patients with autism spectrum disorders. Am J Med Genet B Neuropsychiatr Genet 2011;156:115-124.

36. McGrew SG, Peters BR, Crittendon JA, Veenstra-Vanderweele J. Diagnostic yield of chromosomal microarray analysis in an autism primary care practice: which guidelines to implement? J Autism Dev Disord 2012;42:1582-1591.

37. Betancur $C$. Etiological heterogeneity in autism spectrum disorders: more than 100 genetic and genomic disorders and still counting. Brain Res 2011;1380:4277.

38. Eichler EE, Zimmerman AW. A hot spot of genetic instability in autism. N Eng/ J Med 2008;359(16):1685-1699.

39. Kumar RA, KaraMohamed S, Sudi J, et al. Recurrent $16 p 11.2$ microdeletions in autism. Hum Mol Genet 2008;17:628-638.

40. Weiss LA, Shen Y, Korn JM, et al.; Autism Consortium. Association between microdeletion and microduplication at 16p11.2 and autism. N Engl J Med 2008;358:667-675

41. Walsh KM, Bracken MB. Copy number variation in the dosage-sensitive $16 p 11.2$ interval accounts for only a small proportion of autism incidence: a systematic review and meta-analysis. Genet Med 2011;13:377-384.

42. Jacquemont ML, Sanlaville D, Redon R, et al. Array-based comparative genomic hybridisation identifies high frequency of cryptic chromosomal rearrangements in patients with syndromic autism spectrum disorders. J Med Genet 2006:43:843-849.

43. International Standards for Cytogenomics Arrays Consortium. https://www. iscaconsortium.org.

44. UCSC Genome Bioinformatics. http://genome.ucsc.edu.

45. DECIPHER v5.1. http://decipher.sanger.ac.uk.

46. Database of Genomic Variants. http://projects.tcag.ca/variation.

47. Hatton DD, Sideris J, Skinner M, et al. Autistic behavior in children with fragile $\mathrm{X}$ syndrome: prevalence, stability, and the impact of FMRP. Am J Med Genet $A$ 2006;140A:1804-1813 
48. Watson MS, Leckman JF, Annex B, et al. Fragile X in a survey of 75 autistic males. NEngl J Med 1984;310:1462.

49. Cohen IL, Sudhalter V, Pfadt A, Jenkins EC, Brown WT, Vietze PM. Why are autism and the fragile-X syndrome associated? Conceptual and methodological issues. Am J Hum Genet 1991;48:195-202.

50. Hammer S, Dorrani N, Dragich J, Kudo S, Schanen C. The phenotypic consequences of MECP2 mutations extend beyond Rett syndrome. Ment Retard Dev Disabil Res Rev 2002;8:94-98.

51. Vourc'h P, Bienvenu T, Beldjord $C$, et al. No mutations in the coding region of the Rett syndrome gene MECP2 in 59 autistic patients. Eur J Hum Genet 2001;9:556-558.

52. Beyer KS, Blasi F, Bacchelli E, Klauck SM, Maestrini E, Poustka A; International Molecular Genetic Study of Autism Consortium (IMGSAC). Mutation analysis of the coding sequence of the MECP2 gene in infantile autism. Hum Genet 2002;111:305-309.

53. Carney RM, Wolpert CM, Ravan SA, et al. Identification of MeCP2 mutations in a series of females with autistic disorder. Pediatr Neurol 2003;28:205-211.

54. Zappella M, Meloni I, Longo I, et al. Study of MECP2 gene in Rett syndrome variants and autistic girls. Am J Med Genet B Neuropsychiatr Genet 2003;119B:102-107.

55. Lam CW, Yeung $\mathrm{WL}, \mathrm{Ko} C \mathrm{H}$, et al. Spectrum of mutations in the MECP2 gene in patients with infantile autism and Rett syndrome. J Med Genet 2000;37:E41

56. Herman GE, Henninger N, Ratliff-Schaub K, Pastore M, Fitzgerald S, McBride KL. Genetic testing in autism: how much is enough? Genet Med 2007:9:268-274.

57. Schaefer GB, Lutz RE. Diagnostic yield in the clinical genetic evaluation of autism spectrum disorders. Genet Med 2006;8:549-556.

58. Young DJ, Bebbington $A$, Anderson A, et al. The diagnosis of autism in a female: could it be Rett syndrome? Eur J Pediatr 2008;167:661-669.

59. Psoni S, Sofocleous C, Traeger-Synodinos J, Kitsiou-Tzeli S, Kanavakis E, Fryssira-Kanioura $\mathrm{H}$. Phenotypic and genotypic variability in four males with MECP2 gene sequence aberrations including a novel deletion. Pediatr Res 2010:67:551-556.

60. Ramocki MB, Peters SU, Tavyev YJ, et al. Autism and other neuropsychiatric symptoms are prevalent in individuals with $\mathrm{MeCP} 2$ duplication syndrome. Ann Neurol 2009;66:771-782.

61. Goffin A, Hoefsloot LH, Bosgoed E, Swillen A, Fryns JP. PTEN mutation in a family with Cowden syndrome and autism. Am J Med Genet 2001;105:521-524.
62. Stein MT, Elias ER, Saenz M, Pickler L, Reynolds A. Autistic spectrum disorder in a 9-year-old girl with macrocephaly. J Dev Behav Pediatr 2010:31:632-634.

63. Varga EA, Pastore M, Prior T, Herman GE, McBride KL. The prevalence of PTEN mutations in a clinical pediatric cohort with autism spectrum disorders, developmental delay, and macrocephaly. Genet Med 2009;11:111-117.

64. McBride KL, Varga EA, Pastore MT, et al. Confirmation study of PTEN mutations among individuals with autism or developmental delays/mental retardation and macrocephaly. Autism Res 2010;3:137-141.

65. Buxbaum JD, Cai G, Chaste P, et al. Mutation screening of the PTEN gene in patients with autism spectrum disorders and macrocephaly. Am J Med Genet B Neuropsychiatr Genet 2007;144B:484-491.

66. Zecavati N, Spence SJ. Neurometabolic disorders and dysfunction in autism spectrum disorders. Curr Neurol Neurosci Rep 2009;9:129-136.

67. Baieli S, Pavone L, Meli C, Fiumara A, Coleman M. Autism and phenylketonuria. J Autism Dev Disord 2003;33:201-204.

68. Wraith JE, Danks DM, Rogers JG. Mild Sanfilippo syndrome: a further cause of hyperactivity and behavioural disturbance. Med J Aust 1987;147:450451.

69. Haas RH. Autism and mitochondrial disease. Dev Disabil Res Rev 2010;16:144153.

70. Weissman JR, Kelley RI, Bauman ML, et al. Mitochondrial disease in autism spectrum disorder patients: a cohort analysis. PLOS ONE 2008;3:e3815.

71. Wilder RT, Flick RP, Sprung J, et al. Early exposure to anesthesia and learning disabilities in a population-based birth cohort. Anesthesiology 2009;110:796804.

72. Sprung J, Flick RP, Katusic SK, et al. Attention-deficit/hyperactivity disorder after early exposure to procedures requiring general anesthesia. Mayo Clin Proc 2012;87:120-129.

73. Boddaert N, Zilbovicius M, Philipe A, et al. MRI findings in 77 children with nonsyndromic autistic disorder. PLOS ONE 2009;4:e4415.

74. Schaefer GB, Mendelsohn NJ. Genetics evaluation for the etiologic diagnosis of autism spectrum disorders. Genet Med 2008;10:4-12.

75. Mefford HC, Batshaw ML, Hoffman EP. Genomics, intellectual disability, and autism. N Engl J Med 2012;366:733-743.

76. Schaefer GB, Mendelsohn NJ; Professional Practice and Guidelines Committee. Clinical genetics evaluation in identifying the etiology of autism spectrum disorders. Genet Med 2008;10:301-305. 